\title{
Treatment of Metarhizium fungal keratitis in the United Kingdom
}

\author{
David J. Gunn ${ }^{1} \cdot$ Shokufeh Tavassoli ${ }^{1} \cdot$ Kieren Darcy $^{1}$
}

Received: 5 April 2018 / Accepted: 5 April 2018 / Published online: 11 June 2018

(c) The Royal College of Ophthalmologists 2018

\section{Introduction}

A 22-year-old female patient presented with pain and reduced vision in her right eye for the past few days. She had a three-year history of monthly soft contact lens wear and a past medical history of anxiety managed with citalopram. She was otherwise well. The patient worked as a veterinary student, reported to showering in her contact lenses and denied a history of trauma. On presentation right CDVA was 20/120 with spectacles (pinhole 20/30) and 20/ 16 on the left. The conjunctiva was injected on the right. In the mid peripheral cornea there was a $4 \mathrm{~mm}$ by $4 \mathrm{~mm}$ epithelial defect within which there was a dense infiltrate measuring $2.2 \mathrm{~mm}$ by $1.7 \mathrm{~mm}$. The anterior chamber was quiet. A diagnosis of microbial contact lens related keratitis was made.

A corneal scrape was performed, and levofloxacin $0.3 \%$ drops were started hourly day and night. On review day three, right CDVA had improved to 20/80 (pinhole 20/30) and the eye felt more comfortable. However, the infiltrate and epithelial defect had increased in size. Cefuroxime 5\% drops were added hourly day and night. On review day 5, the visual acuity had improved to $20 / 40$ on the right with glasses (pinhole 20/30). The right eye remained intermittently sore. On examination, the epithelial defect had increased in size to $8 \mathrm{~mm}$ by $8 \mathrm{~mm}$, with the mid peripheral infiltrate now chalky in appearance measuring $2.5 \mathrm{~mm}$ by $2.5 \mathrm{~mm}$. Satellite lesions were also apparent. A clinical diagnosis of fungal keratitis was made, which was confirmed by a positive culture of scanty growth of fungus from the initial corneal scrape.

The patient was admitted for treatment with voriconazole $1 \%$ hourly day and night, along with cefuroxime and

David J. Gunn

drdavidgunn@gmail.com

1 Bristol Eye Hospital, University Hospitals Bristol NHS Foundation Trust, Bristol, UK levofloxacin two hourly. Comfort improved on this treatment and the voriconazole was reduced to hourly day only. The patient was discharged on this treatment at day 7. On day 12 , the right eye started to deteriorate with increased pain. Visual acuity was reduced to $20 / 200$ on the right (pinhole 20/120). Clinical examination revealed a large endothelial plaque behind the infiltrate that had now enlarged to $3 \mathrm{~mm}$ vertical by $2.5 \mathrm{~mm}$ horizontal. A further corneal scrape was performed. This and the original scrape identified Metarhizium species.

Treatment was changed to topical natamycin 5\% hourly day and night. The corneal ulcer and infiltrate improved rapidly on this treatment and it was tapered to two hourly by day 18 . The infiltrate had cleared by day 22 . Treatment was reduced to six times a day and continued with the levofloxacin four times a day. At review day 29, visual acuity was 20/80 on the right (pinhole 20/40) with full resolution of the epithelial defect. The right eye was however mildly injected and keratic precipitates were noted on the endothelium. Topical cyclosporin A $0.1 \%$ was commenced twice daily with natamycin continued six times daily.

At review day 60, right CDVA was 20/20 with glasses and the eye was comfortable. Clinically there was a $3.5 \mathrm{~mm}$ by $3.5 \mathrm{~mm}$ scar, not affecting the visual axis. The eye was white, with no epithelial defect or infiltrate and treatment was ceased. The patient was reviewed on day 75 and remained asymptomatic off all treatment with a CDVA of $20 / 20$ and was discharged.

\section{Discussion}

Metarhizium anispliae is a filamentous entomopathogenic fungus used worldwide as an agricultural insecticide [1]. The organism has optimal growth at $25^{\circ} \mathrm{C}$ and as such has been considered safe, though has extremely rarely being implicated in human disease [1,2]. Metarhizium has been implicated in a small number of cases of keratitis, sclerokeratitis and endophthalmitis. This report to our knowledge is the ninth worldwide for an ocular infection. The first 


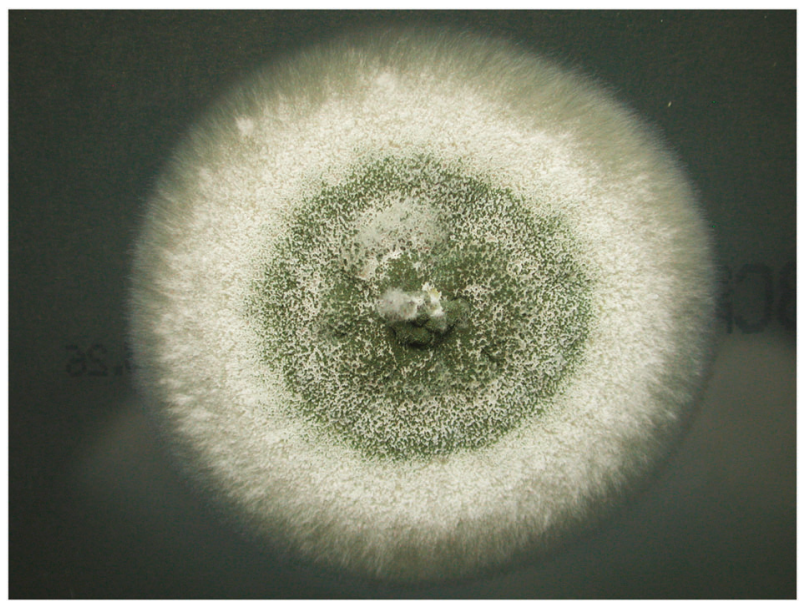

Fig. 1 Macroscopic appearance of Metarhizium species identified

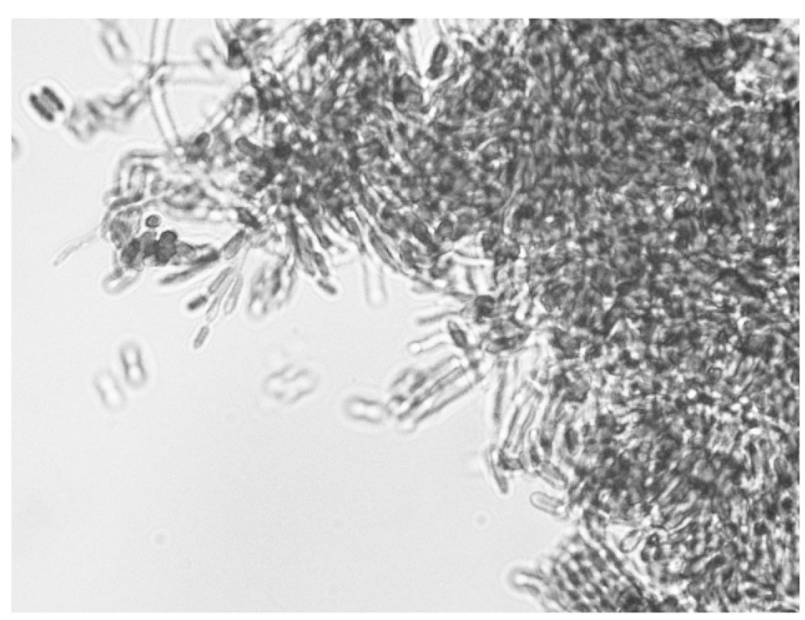

Fig. 2 Microscopic appearance of Metarhizium species identified

Table 1 Antifungal susceptibility reports from three Metarhizium keratitis case reports. MIC presented in $\mathrm{mg} / \mathrm{L}$

\begin{tabular}{|c|c|c|c|}
\hline Reference & This report & $\begin{array}{l}\text { Eguchi et al. } \\
\text { [7] }\end{array}$ & $\begin{array}{l}\text { Dorin et al. } \\
\text { [2] }\end{array}$ \\
\hline $\begin{array}{l}\text { Susceptibility } \\
\text { method }\end{array}$ & $\begin{array}{l}\text { CLSI broth } \\
\text { dilution }\end{array}$ & $\begin{array}{l}\text { CLSI broth } \\
\text { dilution }\end{array}$ & $E$-test $\mathrm{R}$ \\
\hline Amphotericin B & Resistant $[m=2]$ & Resistant & Resistant \\
\hline Voriconazole & Sensitive $[m=1]$ & Sensitive & Sensitive \\
\hline Econazole & $\begin{array}{l}\text { Intermediate }[\mathrm{m} \\
=2]\end{array}$ & - & - \\
\hline Itraconazole & $\begin{array}{l}\text { Resistant }[m> \\
16]\end{array}$ & Sensitive & Resistant \\
\hline Natamycin & Sensitive $[m=8]$ & - & - \\
\hline Posaconazole & - & - & Sensitive \\
\hline Fluconazole & - & Sensitive & Resistant \\
\hline Miconazole & - & Sensitive & - \\
\hline Micafungin & - & Sensitive & Sensitive \\
\hline Caspofungin & - & - & Sensitive \\
\hline Flucytosine (5-FC) & - & Resistant & Resistant \\
\hline
\end{tabular}

report was in 1997 from Colombia [3], then subsequent reports emerged from the United States [4, 5], Australia [6], Japan [7], France [2, 8] and Canada [9]. This is the first report in the United Kingdom. Of the previous eight cases, four implicated contact lens wear [4, 5, 7, 9], three involved ocular injection of organic material [2, 6, 8] whilst the initially described case had no identifiable risk factors [3].

Corneal scrape samples were cultured, and fungal elements identified at the North Bristol Bacteriology laboratory. Isolates were sent to the Public Health England South West Mycology Reference Laboratory for identification. The organism was identified as Metarhizium species. Macroscopic and microscopic images are shown in Figs. 1 and 2. Susceptibility studies were performed by CLSI (Clinical Laboratory Standards Institute) broth microdilution method M38-A2. Results are presented in Table 1 with those from the only other two ocular cases with reported susceptibilities [2, 7].

Amphotericin-B resistance has been almost universal in ocular and non-ocular Metarhizium mycosis susceptibility reports, indicating a possible intrinsic resistance to the agent [2]. Despite in vitro sensitivity to voriconazole in the reported susceptibilities, there was little response in our case with clinical deterioration. A similar trend was seen with progression despite voriconazole therapy delivered topically [9] or systemically [2] in other cases. It is established that for filamentous fungi particularly, in vitro MIC values indicating susceptibility do not always correlate with effective host response to treatment [10].

Natamycin was used to successfully treat three previous cases with excellent outcomes [3-5]. Conversely, two cases progressed to keratoplasty and poor outcome despite natamycin treatment $[6,7]$. In the two cases where natamycin was unavailable, infection progressed to require therapeutic keratoplasty despite topical and systemic antifungals, one case with corneal perforation at day 11 of treatment [2, 9].

Natamycin $5 \%$ is employed as a first line empiric antimycotic therapy in many centres [10]. Potential drawbacks include limited ocular penetration, lack of widespread availability of a commercial ophthalmic product and restricted efficacy in yeast type keratomycoses. Empiric first-line treatment with natamycin $5 \%$ is optimal for superficial mycotic appearing infiltrates or those with filamentous elements identified on microscopy or culture [10]. In the context of Metarhizium species identification, this case adds support to the use of topical natamycin. This report further demonstrates clear clinical inferiority of voriconazole despite in vitro sensitivity as well as the presence of amphotericin B resistance in Metarhizium keratitis samples.

Acknowledgements Thanks to Dr Elizabeth Johnson and Adrien Szekely for Metarhizium images included in Figs. 1 and 2. 


\section{Compliance with ethical standards}

Conflict of interest The authors declare that they have no conflict of interest.

\section{References}

1. Roberts DW, St Leger RJ. Metarhizium spp., cosmopolitan insectpathogenic fungi: mycological aspects. Adv Appl Microbiol. 2004;54:1-70.

2. Dorin J, Debourgogne A, Zaidi M, et al. First unusual case of keratitis in Europe due to the rare fungus Metarhizium anisopliae. Int J Med Microbiol. 2015;305:408-12.

3. De Garcia MC, Arboleda ML, Barraquer F, et al. Fungal keratitis caused by Metarhizium anisopliae var. anisopliae. J Med Vet Mycol. 1997;35:361-3.
4. Jani BR, Rinaldi MG, Reinhart WJ. An unusual case of fungal keratitis: Metarrhizium anisopliae. Cornea. 2001;20:765-8.

5. Motley WW, Melson AT, Mortensen JE. Pediatric Metarrhizium anisopliae keratitis. J AAPOS. 2011;15:101-3.

6. Amiel H, Chohan AB, Snibson GR, et al. Atypical fungal sclerokeratitis. Cornea. 2008;27:382-3.

7. Eguchi H, Toibana T, Hotta F, et al. Severe fungal sclerokeratitis caused by Metarhizium anisopliae: a case report and literature review. Mycoses. 2015;58:88-92.

8. Derhy D, Sauer A, Sabou M, et al. Surgical treatment of Metarhizium anisopliae sclerokeratitis and endophthalmitis. Indian $\mathbf{J}$ Ophthalmol. 2017;65:523-6.

9. Showail MJ, Kus JV, Tsui GK, et al. Fungal keratitis caused by Metarhizium anisopliae complex. Med Mycol Case Rep. 2017;17:28-30.

10. Thomas PA, Kaliamurthy J. Mycotic keratitis: epidemiology, diagnosis and management. Clin Microbiol Infect. 2013;19:210-20.

\title{
Reversible retinal toxicity in early oral Entecavir therapy for hepatitis-B infection
}

\author{
Suman Pilli ${ }^{1} \cdot$ Peck Lin Lip ${ }^{1}$
}

Received: 30 April 2018 / Accepted: 22 May 2018 / Published online: 22 June 2018

(c) The Royal College of Ophthalmologists 2018

We illustrate an acute retinal toxicity from oral Entecavir (Bristol-Myers-Squibb, USA) as treatment for acute hepatitis-B infection, and subsequent reversal of retinal toxicity upon stopping Entecavir with full restoration of visual functions.

\section{Case report}

A 52-year-old Caucasian male presented with unilateral sudden painless reduced vision and central scotoma of 5day duration. Patient was diagnosed acute hepatitis-B infection 6 weeks before and was started with oral

Peck Lin Lip

pllipwoo@gmail.com

1 Birmingham and Midland Eye Centre, Sandwell and West Birmingham Hospitals NHS Trust, City Hospital, Dudley Road, Birmingham B18 7QH, UK
Entecavir $0.5 \mathrm{mg}$ once daily. His presenting vision was 6/15OS and 6/6OD, normal pupillary reactions, full colour-perception and no ocular inflammation. Right fundus was normal while left showed mildly hyperaemic optic disc and a central well-demarcated hypopigmented area in posterior pole (Fig. 1: 1A). Both fundusfluorescein angiography (Fig. 1) and optical coherence tomography (Fig. 2) depicted corresponding retinal pigment epithelial (RPE) and photoreceptors abnormality. He had also established patchy central Goldmann field defects and grossly reduced full-field electroretinogram (delayed responses from both rod and cones) in OS only. Excluded retinovasculitis and satisfactory normal systemic investigations, a suspicion of drug toxicity was made. Entecavir was stopped immediately and remained off when his liver function test was also normalised. At 2month review, his vision had reached $6 / 6$, centre RPE hypopigmentation became indistinct. By 5 months, all visual function tests and fundoscopy were fully restored (Figs. 1, and 2). 

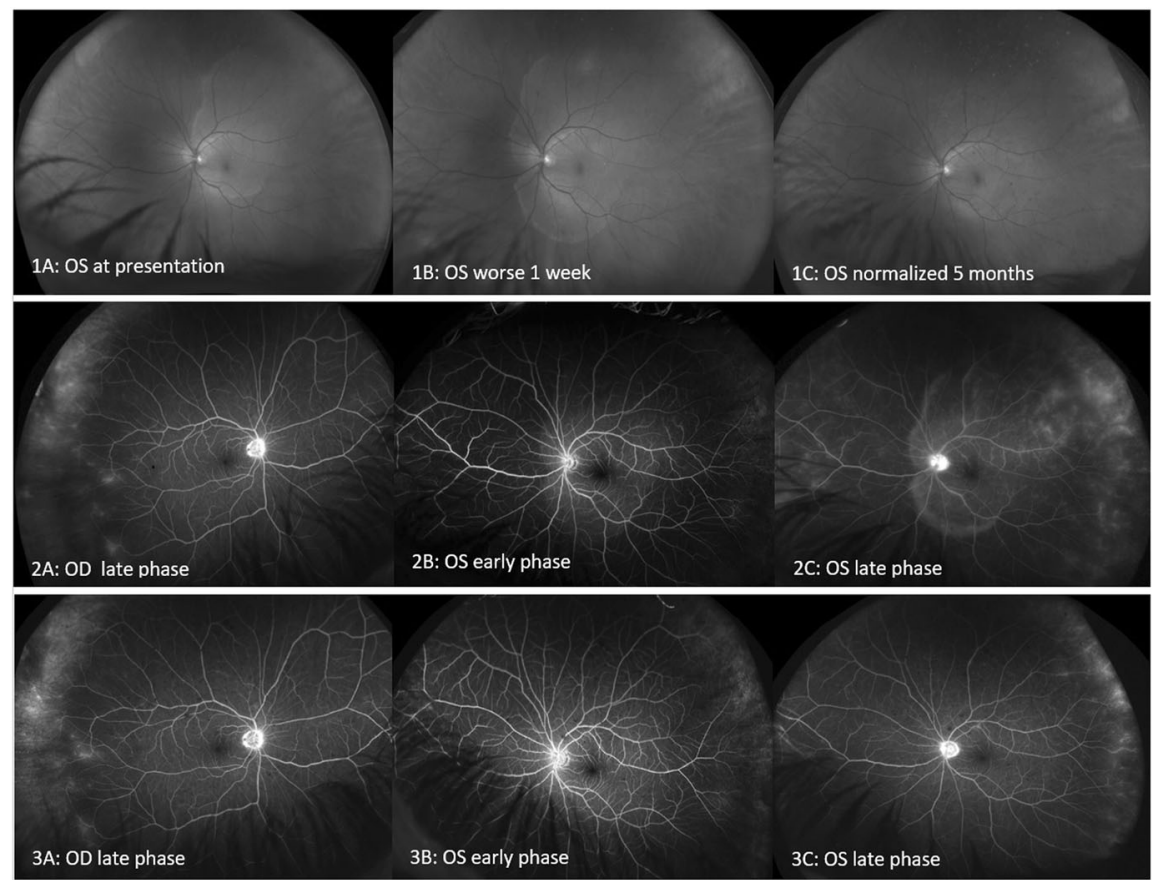

Fig. 1 Fundus photographs and fluorescein angiography (FFA). Fundus photographs of OS showed a clear demarcation of retinal pigment epithelial (RPE) hypopigmentation in posterior pole at presentation (1A). Patient's vision was 6/15 and complained of central scotoma, Entecavir was stopped at this point due to suspicion of retinal toxicity. At 1-week review, vision was worse (6/18 OS) and there was evidence of enlargement in demarcated RPE hypopigmentation area (1B). At 5-month review, patient had made a full spontaneous visual recovery in all aspects with 6/6 vision (1C). FFA at 1 week after presentation $(2 \mathrm{~A}-\mathrm{C})$ : OD showed late-phase temporal peripheral distal venous staining and peripapillary hyperfluorescence but normal posterior pole (2A). OS showed early well-demarcated area of early RPE layer hyperfluorescence corresponding to clinical hypopigmentation (2B), and remained staining in late phase (2C). There was more intense hyperfluorescence on optic disc and wider peripheral distal venous staining. FFA at 5 months after presentation $(3 \mathrm{~A}-\mathrm{C})$ : both eyes showed significant normalisation with some residual peripheral venous staining. $\mathrm{OS}=$ Left eye, $\mathrm{OD}=$ Right eye

Fig. 2 Optical coherence tomography (OCT) scans. At presentation, OCT showed normal retinal layers in OD (a), contrasting abnormal retinal layers in OS (b) with loss of reflectivity of photoreceptor ellipsoid zone and outer segments with intact external limiting membrane (white arrowhead), and multiple small high reflective elevations seen on RPE layer (white arrows). Repeated OCT at 5 months confirmed restoration of all retinal layers in OS (c). $\mathrm{OS}=$ Left eye, $\mathrm{OD}=$ Right eye

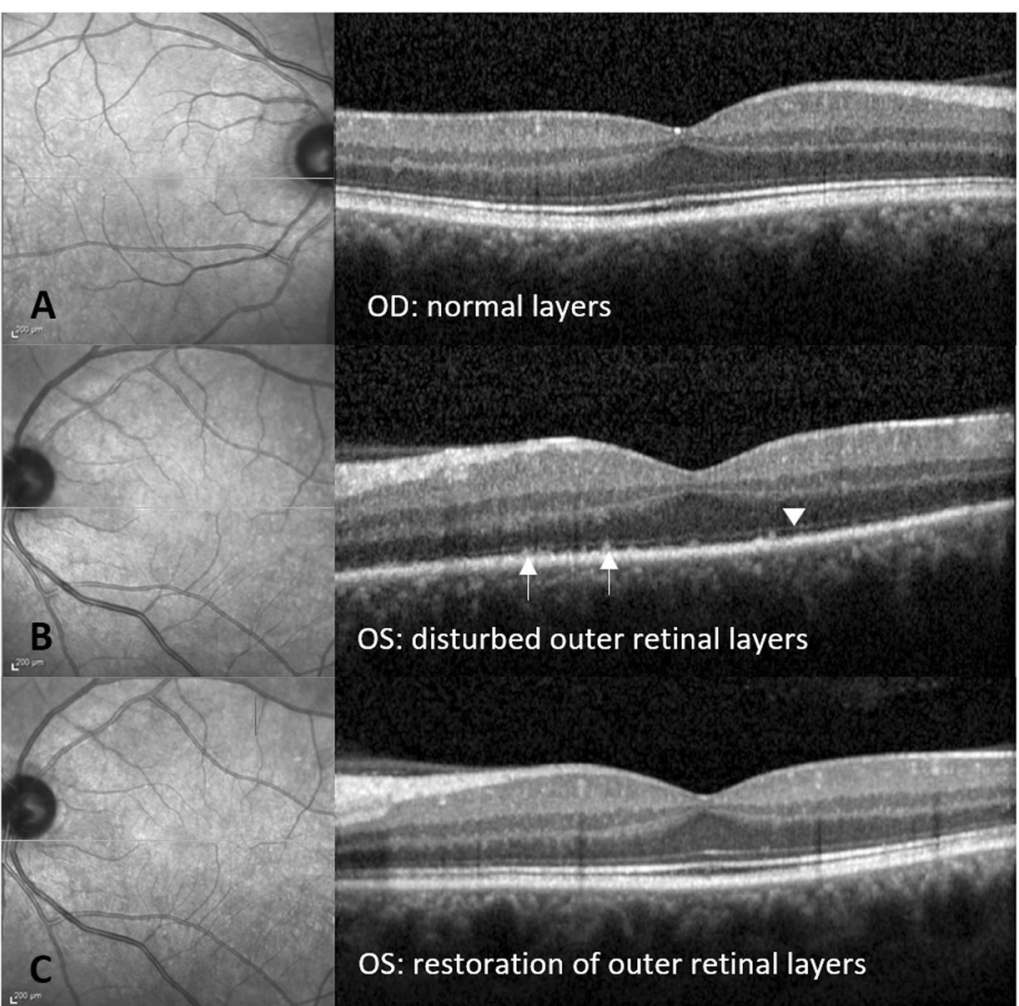


Entecavir is an anti-viral drug commonly used in treatment of hepatitis-B infection but there was no recognised ocular toxicity in its association [1-3]. The mechanism of retinal toxicity remains unclear with lacking histopathological studies. There was a published case report with irreversible blindness when on year(s) of Entecavir complicated by diabetic retinopathy at the time of suspected retinal toxicity [4]. Our case shared similar clinical features as their reported second eye. Notably, Entecavir drug toxicity is causing sequential rather than bilateral simultaneous eye manifestations. Our patient's left eye was profoundly involved but his right eye had not progressed more than the minimal peripheral vascular staining in late fundus photographs and fluorescein angiography phase (Fig. 1: 2A).

Our case confirmed short-term Entecavir use could cause reversible retinal toxicity upon drug cessation. Prescribing physicians and ophthalmologists should be aware of the potential retinal toxicity of Entecavir. We reported this adverse drug reaction to the UK Medicines and Health Products Regulatory Agency.
Acknowledgements We thanked Dr. Peter Good/Dr. Emma Berrow with their visual function team and Ms. Rosie Auld with her orthoptic team for their accommodative prompt services in performing/interpreting the results of visual function tests at various stages.

\section{Compliance with ethical standards}

Conflict of interest The authors declare that they have no conflict of interest.

\section{References}

1. Entecavir. National Center for Biotechnology Information. Pubchem compound database; $\mathrm{CID}=153941$. http://pubchem.ncbi.nlm. nih.gov/compound/153941.

2. Wong GL, Seto WK, Wong VW, Yuen MF, Chan HL. Review article: long-term safety of oral anti-viral treatment for chronic hepatitis-B. Aliment Pharmacol Ther. 2018;47:730-7.

3. Kayaaslan B, Guner R. Adverse effects of oral antiviral therapy in chronic hepatitis B. World J Hepatol. 2017;9:227-41.

4. Muqit MK, Stanga PE, Vilar FJ, Patton N. Presumed entecavirinduced ocular toxicity. Eye. 2011;25:1665-8.

\title{
Christmas tree cataract and myotonic dystrophy type 1
}

\author{
Dionysios Pagoulatos ${ }^{1} \cdot$ Zoi Kapsala $^{2} \cdot$ Olga E. Makri $^{1} \cdot$ Constantinos D. Georgakopoulos $^{1}$
}

Received: 14 March 2018 / Revised: 2 May 2018 / Accepted: 1 June 2018 / Published online: 9 July 2018

(c) The Royal College of Ophthalmologists 2018

We have read with great interest the recent article by Papadopoulos et al. [1] reporting early onset posterior subscapular cataract in 9 out of 28 patients with myotonic dystrophy type 2 (DM2). They also mentioned that this type of cataract was the first symptom in $7(25 \%)$ of the studied DM2 patients.

Dionysios Pagoulatos pagdion@gmail.com

1 Department of Ophthalmology, School of Medicine, University of Patras, Patras, Greece

2 Department of Ophthalmology, School of Medicine, University of Crete, Heraklion, Greece
Here, we would like to present our results regarding the incidence of Christmas tree cataract in patients with the most common form of DM, myotonic dystrophy type 1 (DM1). Christmas tree cataract is considered a characteristic finding in subjects with DM1. The retrospective review of medical records of 23 patients with DM1 revealed the presence of Christmas tree cataract in 13 patients $(56 \%)$. The multicoloured, iridescent lens opacities were unilateral in 10 out of the 13 patients and asymmetric bilateral in 3 patients. Age when cataract was diagnosed was $47 \pm 5$ years (range: $35-52$ years). The cataract was the first sign of the disease for 11 patients and was detected accidentally during a routine ophthalmological examination. Best corrected visual acuity was $0.06 \pm 0.08 \log$ MAR (range: -0.1 to $0.2 \operatorname{logMAR}$ ). The interval between diagnosis of cataract and DM1 was $10 \pm 2$ years (range: 
7-14 years). This delay is explained by the fact that none of the patients presented typical symptoms of DM1 at the time of the diagnosis of the cataract. Remarkably, apart from Christmas tree cataract, no other form of cataract was detected in the study group, as well as no other pathology regarding the anterior or posterior segment was recorded.

Notably, in previous studies, Christmas tree cataract has been detected in nearly all patients with DM1, and viceversa $16.7 \%$ of subjects with Christmas tree cataract have been diagnosed with DM1 [2].

In conclusion, earlier detection of Christmas tree cataract, which constitutes a common ophthalmologic finding in DM1 patients [3, 4], and thus, referral for neuromuscular assessment could possibly eliminate delays in diagnosis of the disease. Noteworthy, it seems that Christmas cataract may be the first manifestation of DM1 in a higher percentage of patients than posterior subcapsular cataract in DM2 patients. Extended multicenter studies may elucidate the exact incidence of the different cataract types in these patients.

\section{Compliance with ethical standards}

Conflict of interest The authors declare that they have no conflict of interest.

Informed consent Written informed consent was obtained from all patients and the study was approved by the institutional review board at the University of Patras.

\section{References}

1. Papadopoulos C, Kekou K, Xirou S, Kitsiou-Tzeli S, Kararizou E, Papadimas GK. Early onset posterior subscapular cataract in a series of myotonic dystrophy type 2 patients. Eye (Lond). 2018;32:622-625

2. Reiter C, Gramer E. Anticipation in patients with iridescent multicoloured posterior capsular lens opacities ("Christmas tree cataract"): the role in the diagnosis of myotonic dystrophy. Ophthalmologe. 2009;106:1116-20.

3. Bird TD. Myotonic dystrophy type 1. In: Adam MP, Ardinger HH, Pagon RA, et al., editors. GeneReview [Internet]. Seattle, WA: University of Washington; Seattle; 1999. p. 1993-2017.

4. Stival L, Bittar R, Lago A, Nassaralla J Jr. Christmas tree cataract. Rev Bras Oftalmol. 2015;74:309-11.

\title{
Hypothyroidism and non-infectious uveitis
}

\author{
Shorya Vardhan Azad ${ }^{1} \cdot$ Varun Gogia $^{1} \cdot$ Pradeep Venkatesh $^{1} \cdot$ Brijesh Takkar $^{1}$
}

Received: 7 February 2018 / Accepted: 12 February 2018 / Published online: 24 July 2018

(c) The Royal College of Ophthalmologists 2018

Understanding the non-specific symptoms of uveitis and its corroboration with systemic findings may give insight into its pathophysiology and may in turn help in its management. Association of thyroid disorders with uveitis is well known; however, emphasis has not been made in most of the past studies regarding its prevalence and spectrum of presentation $[1,2]$.

We performed a prospective analysis of patients presenting at our uvea clinic at a tertiary eye care center in Northern India and evaluated the patterns of uveitis in our

Pradeep Venkatesh

venkyprao@yahoo.com

1 Dr. Rajendra Prasad Centre for Ophthalmic Sciences, AIIMS, New Delhi, India population. As a part of study we also analyzed the prevalence of associated systemic diseases and systemic symptoms. We observed that $24.5 \%$ of patients of uveitis had underlying systemic disease, where past or present evidence of tuberculosis was most common followed by seronegative spondyloarthropathy and sarcoidosis with overall prevalence of $6.8 \%, 4.1 \%$, and $2.5 \%$, respectively. Prevalence of hypothyroidism in these patients was $1.5 \%$ amongst (12 patients out of 800 ) all patients and 6.1\% among patients with known systemic illness. There were 10 females and 2 males with median age of 37 years (Table 1). Spectrum of involvement was varied, with intermediate uveitis (IU) being the most common presentation. All patients had confirmed hypothyroidism and were on thyroid replacement therapy. None of them suffered from hyperthyroidism. Thyroid functions tests and anti-thyroid 
Table 1 Demographic features, laterality and anatomical involvement in patients with thyroid dysfunction and uveitis

\begin{tabular}{|c|c|c|c|c|}
\hline $\begin{array}{l}\text { Age of } \\
\text { patient } \\
\text { (years) }\end{array}$ & Gender & $\begin{array}{l}\text { Laterality of } \\
\text { the disease }\end{array}$ & $\begin{array}{l}\text { Anatomical } \\
\text { classification of } \\
\text { uveitis }\end{array}$ & $\begin{array}{l}\text { Systemic } \\
\text { symptoms }\end{array}$ \\
\hline 20 & Male & Unilateral & Posterior & Joint pains \\
\hline 40 & Female & Bilateral & Intermediate & None \\
\hline 33 & Female & Unilateral & Intermediate & None \\
\hline 32 & Female & Bilateral & Anterior & None \\
\hline 35 & Female & Bilateral & Anterior & None \\
\hline 36 & Male & Unilateral & Intermediate & None \\
\hline 38 & Female & Bilateral & Panuveitis & None \\
\hline 52 & Female & Bilateral & Anterior & None \\
\hline 40 & Female & Unilateral & Intermediate & None \\
\hline 42 & Female & Bilateral & Intermediate & None \\
\hline 42 & Female & Unilateral & Intermediate & Joint pains \\
\hline 33 & Female & Unilateral & Anterior & None \\
\hline
\end{tabular}

antibodies were not ordered at the time of presentation as all patients were already on treatment.

Numerous studies have described systemic associations of uveitis, however, only a few studies have reported such an association between thyroid dysfunction and uveitis [3, 4]. Also, none of them have assessed the prevalence and type of uveitis. A previous study that investigated the relationship between thyroid dysfunction and uveitis, concluded that hypothyroidism was a more frequent association [1]. Our analysis echoed similar results with none of the patients having hyperthyroidism. However, aforementioned study tested thyroxine levels in all patients with or without a specific etiological diagnosis whereas our study included only patients with an established diagnosis of hypothyroidism by an endocrinologist. When compared to prevalence of hypothyroidism in community, nearly $10 \%$ of normal population also have subclinical hypothyroidism [5]. Hence, the data described here might be an underestimation of associated hypothyroidism because we did not order thyroid function tests in other idiopathic uveitis which is a major limitation.

Another important component of our analysis was the anatomical category of uveitis. This revealed IU as being the most common presentation (50\%). Similar study evaluating systemic associations in IU, showed an overall prevalence of thyroid dysfunction at $6.02 \%$ among all patients of IU and $19.23 \%$ amongst patients with known systemic illness [2]. In their study 3 patients had hypothyroidism. Although we cannot decipher a direct causal relationship of thyroid abnormalities and uveitis, concurrent systemic disease may play a role in predisposition for the same.

In conclusion, we highlight surprisingly frequent prevalence of thyroid dysfunction in patients with noninfectious uveitis and its varied presentations. Routine evaluation of thyroid function and related antibodies in cases of idiopathic uveitis may likely seem justified and may reveal a higher association.

\section{Compliance with ethical standards}

Conflict of interest The authors declare that they have no conflict of interest.

\section{References}

1. Cantor LB, Weber JC, Schlaegel TF. Thyroid dysfunction and uveitis. Ann Ophthalmol. 1982;14:515-7.

2. Boskovich SA, Lowder CY, Meisler DM, Gutman FA. Systemic diseases associated with intermediate uveitis. Cleve Clin J Med. 1993;60:460-5.

3. Rathinam SR, Namperumalsamy P. Global variation and pattern changes in epidemiology of uveitis. Indian $\mathrm{J}$ Ophthalmol. 2007;55:173-83.

4. Das D, Bhattacharjee H, Bhattacharyya PK, Jain L, Panicker MJ, Das K, et al. Pattern of uveitis in North East India: a tertiary eye care center study. Indian J Ophthalmol. 2009;57:144-6.

5. Usha Menon V, Sundaram KR, Unnikrishnan AG, Jayakumar RV, Nair V, Kumar H. High prevalence of undetected thyroid disorders in an iodine sufficient adult south Indian population. J Indian Med Assoc. 2009;107:72-7. 